\title{
Probing the Structure of Ribosome Assembly Intermediates In vivo using DMS and Hydroxyl Radical Footprinting
}

\author{
${ }^{1}$ Ryan M. Hulscher, ${ }^{2}$ Jen Bohon, ${ }^{1}$ Mollie C. Rappé, ${ }^{3}$ Sayan Gupta, ${ }^{2}$ Rhijuta D’Mello, \\ ${ }^{2}$ Michael Sullivan, ${ }^{3}$ Corie Y. Ralston, ${ }^{2}$ Mark R. Chance, ${ }^{1}$ Sarah A. Woodson*
}

\begin{abstract}
${ }^{1}$ T. C. Jenkins Department of Biophysics, Johns Hopkins University, 3400 N. Charles St., Baltimore, MD 21218 USA

${ }^{2}$ Center for Proteomics and Bioinformatics and Center for Synchrotron Biosciences, Case Western Reserve University, Upton, New York 11973 USA

${ }^{3}$ Molecular Biophysics and Integrated Bioimaging, Lawrence Berkeley National

Laboratory, Berkeley, California 94720, USA
\end{abstract}

*Correspondence to swoodson@jhu.edu; Tel. 410-516-2015; FAX 410-516-4668 


\begin{abstract}
The assembly of the E. coli ribosome has been widely studied and characterized in vitro. Despite this, ribosome biogenesis in living cells is only partly understood because assembly is coupled with transcription, modification and processing of the preribosomal RNA. We present a method for footprinting and isolating pre-rRNA as it is synthesized in E. coli cells. Pre-rRNA synthesis is synchronized by starvation, followed by nutrient upshift. RNA synthesized during outgrowth is metabolically labeled to facilitate isolation of recent transcripts. Combining this technique with two in vivo RNA probing methods, hydroxyl radical and DMS footprinting, allows the structure of nascent RNA to be probed over time. Together, these can be used to determine changes in the structures of ribosome assembly intermediates as they fold in vivo.
\end{abstract}




\section{Introduction}

Ribonucleoprotein (RNP) complexes typically undergo many structural changes during their lifecycles, from transcription of the RNA to maturation of the complex and degradation. Because the full lifecycle of an RNP cannot often be reconstituted in the test tube, footprinting methods for probing RNA structure in the cell have gained increasing attention [1,2]. Here, we describe methods for probing the structure of the $16 \mathrm{~S}$ ribosomal RNA in E. coli cells following metabolic labeling of pre-rRNA. This approach can be adapted to high-throughput investigations of RNP dynamics.

The bacterial ribosome is made up of three rRNAs and over 50 proteins that assemble into the large (50S) and small (30S) subunits. Assembly of the subunits is coupled with pre-rRNA synthesis [3-5] and requires only a few minutes during logarithmic growth [6]. Near the end of the assembly process, an intermediate 17S form of the pre-rRNA is trimmed to its mature $(16 \mathrm{~S})$ length in several steps by ribonucleases. Several features likely contribute to the rapidity of subunit biogenesis in the cell. First, the 5'-to-3' polarity of co-transcriptional assembly limits the opportunities of forming non-native RNA secondary structures, because 5' regions of the rRNA are able to fold before the 3' regions have been transcribed [5]. Second, more than 15 assembly factors and additional RNA and protein modification enzymes facilitate assembly and carry out the final steps of subunit maturation $[7,8]$. Third, homeostasis of free ribosomal proteins ensures a constant pool of protein components [9]. In eukaryotes, ribosome assembly is aided by over 200 assembly factors and is also coupled to transport of assembly intermediates from the site of transcription in the nucleolus to the cytoplasm [10]. 
Because the bacterial ribosomal subunits can be reconstituted in vitro using only free rRNA and ribosomal proteins [11,12], they have long served as a model for the assembly of ribonucleoprotein complexes. Despite this, ribosome biogenesis in living cells remains poorly understood. One reason for this is the low abundance of assembly intermediates, which constitute $2-5 \%$ of total rRNA in E. coli under normal growth conditions [6]. Moreover, the predominant intermediates are difficult to isolate using sucrose gradient sedimentation, because they tend to migrate near the mature subunits.

Previous studies increased the concentration of ribosomal intermediates by using temperature-sensitive strains or conditional mutations that stall assembly under nonpermissive conditions such as low temperature [13]. Ideally, one should study the path of assembly in real time, under normal conditions. The kinetics of ribosome synthesis or protein binding has been measured by pulse-labeling cells with isotopically-modified nucleosides or amino acids and then analyzing via scintillation counting [6] or mass spectrometry [14]. These approaches reveal the protein composition of assembly intermediates. When complemented by structure probing of the RNA, a more complete picture of the assembly process can be obtained.

We present here a technique for probing the structure of ribosome assembly intermediates in E. coli (Figure 1). To synchronize pre-rRNA synthesis, we take advantage of the role that two intracellular small molecule effectors play in regulating the initiation of bacterial rRNA transcription. In E. coli, rRNA transcription is repressed during starvation through both the stringent response (ppGpp) and the concentration of the initiating NTP [15]. During the late stationary phase and extended periods of starvation, the concentration of the initiating NTP becomes the primary repressor of $r r n$ 
promoter activity [15]. When nutrients become available, there is a rapid increase in intracellular NTP concentration and pre-rRNA synthesis is reactivated. For log phase cells subject to a nutrient upshift and cells diluted from stationary phase into fresh media, rrn promoter activity increases within $1 \min [15]$.

In order to label nascent transcripts when pre-rRNA transcription resumes, we pulse-labeled starved cells with 4-thiouridine (4sU) just before feeding [16]. After extracting the total RNA from the cells, the nascent RNA labeled with $4 \mathrm{sU}$ can be conjugated to a biotinylation reagent via a reversible disulfide bond [17]. The labeled RNA is captured on streptavidin beads and analyzed by direct primer extension or highthroughput sequencing. In this way, it is possible to get snapshots of the ribosome assembly process after transcription begins again.

$4 \mathrm{sU}$ is photoactive and has been widely used to study RNA-protein complexes by in-cell photocrosslinking $[16,18]$. More recently, metabolic labeling with $4 \mathrm{~s} U$ has been used to isolate RNA from yeast [17] and metazoan cells [19] for transcriptome studies. To our knowledge, this represents the first use of $4 \mathrm{sU}$ to isolate newly synthesized RNA in bacteria.

A variety of reagents, such as dimethylsulfate (DMS), ribonucleases, Nmethylisotoic acid (SHAPE) and hydroxyl radicals, have been used to probe RNA structures in vitro [20,21]. DMS and hydroxyl radical (X-ray) footprinting are particularly useful for probing RNA structures in the cell. DMS is lipophilic and readily passes the cell membrane [22,23]. Hydroxyl radicals can be generated in situ by synchrotron or gamma radiation [24-26]. We have successfully used hydroxyl radicals 
and DMS to probe rRNA in vivo with a time resolution of $30 \mathrm{~s}$. Both techniques will be presented here.

Time-resolved X-ray-dependent hydroxyl radical footprinting has been used to study the kinetics of in vitro RNA folding [27] because sufficient hydroxyl radicals to probe RNA can be generated in a few milliseconds using a synchrotron X-ray source with high flux density [28]. X-ray footprinting has also been used on frozen E. coli cells to characterize the structure of the small subunit of the ribosome and examine the effect of ribosome assembly factor deletion [29]. Because the hydroxyl radical is produced in situ by photolysis of the water, no harmful permeabilization of the cell is needed. Another advantage of X-ray footprinting is that the extent of cleavage can be controlled by varying the X-ray dose [30].

The hydroxyl radicals break the RNA strand by abstracting a proton from the ribose $\mathrm{C}^{\prime}$ ', $\mathrm{C5}^{\prime}$ ' or $\mathrm{C} 1^{\prime}$ [31]. The probability of cleavage depends on the solvent accessibility of the ribose, and thus reflects the RNA tertiary structure or protein interactions. Because hydroxyl radicals cleave the RNA backbone in a base-independent manner, hydroxyl radical footprinting provides structural data on the RNA at singlenucleotide resolution, unlike many other chemical probing techniques.

In contrast to hydroxyl radical, DMS methylates adenine $\mathrm{N} 1$, cytosine $\mathrm{N} 3$ and guanine N7, and therefore the extent of modification reports on the RNA secondary structure and other interactions with the RNA bases. Methylation of A N1 and C N3 is detected by pausing of reverse transcriptase during primer extension. Methylation of $\mathrm{G}$ $\mathrm{N} 7$ is usually only detected by primer extension if it subsequently causes depurination. DMS has been extensively used to probe RNA in vitro [32-34], and because it is highly 
reactive, can also be used to monitor RNA assembly in real time [35]. It has also been used in vivo for transcriptomics studies [36]. High-throughput DMS probing of rRNA structure has been performed in bacteria, yeast, and mammalian cells [36-38]. While DMS only modifies certain bases, it does not require a synchrotron, is reproducible, and easy to scale up.

The protocols below describe X-ray and DMS footprinting of RNA in actively dividing cells in order to map changes in the small ribosomal subunit that take place during assembly. The reader can choose to use either technique or both depending on the resources of the lab and the requirements of the experiment.

\section{Materials and Methods}

\subsection{Overview of Starvation Recovery}

During normal cell growth, the ribosomes being synthesized in the cell are in various states of assembly, giving a time-average of the different pre-rRNA intermediates. To synchronize transcription of the rRNA, we exploited the fact that rRNA transcription in E. coli is repressed during starvation by the stringent response and then activated when nutrients become available [15]. As illustrated in Figure 2, little 17S prerRNA is present in E. coli MRE600 cells suspended in a minimal medium lacking phosphate. Upon addition of a rich medium containing phosphate, $17 \mathrm{~S}$ pre-rRNA is detected within 1-2 min, consistent with the kinetics of pre-rRNA synthesis during recovery from stationary phase [15]. When $4 \mathrm{sU}$ is added to the medium before the food, we recover 4 sU-labeled pre-rRNA with similar kinetics. 


\subsection{Starvation Recovery Protocol}

To prepare cultures, $3-5 \mathrm{~mL}$ of $\mathrm{LB}$ media is inoculated with a single bacterial colony and grown overnight at $37{ }^{\circ} \mathrm{C}$ with shaking. The following day, the culture is diluted at a ratio of 1:100 to 1:1000 into $500 \mathrm{~mL}$ media. After the cells reach stationary phase, they are harvested by centrifugation, and resuspended in a defined minimal medium lacking phosphate (100 mM Tris- $\mathrm{HCl}, 25 \mathrm{mM} \mathrm{KCl,} 10 \mathrm{mM} \mathrm{NaCl}, 20 \mathrm{mM}$ $\mathrm{NH}_{4} \mathrm{Cl}, 1 \mathrm{mM} \mathrm{MgSO}{ }_{4}, 0.1 \mathrm{mM} \mathrm{CaCl}_{2}, 2 \mu \mathrm{M}$ thiamine, $0.4 \%$ glucose, $\mathrm{pH}$ 7.8). The cells are harvested and resuspended twice more to remove excess phosphate. On the final step, minimal media is added until the cells are diluted to the desired final volume and have an $\mathrm{OD}_{600}$ in the mid-log range (0.6-0.8). For our experiments, we use $75 \mathrm{~mL}$ per time course, or $675 \mathrm{~mL}$ for 9 trials. The cells are incubated in minimal media at $37^{\circ} \mathrm{C}$ with shaking for $4 \mathrm{~h}$ to arrest pre-rRNA transcription.

At the end of this starvation period, and approximately 2 minutes before rRNA transcription is induced, a $250 \mathrm{mM}$ stock solution of the modified nucleotide 4thiouridine ( $4 \mathrm{sU})$ is added to the media to a final concentration of $0.5 \mathrm{mM}$. This concentration of $4 \mathrm{sU}$ minimally affects the growth rate of MRE600 (data not shown). The stock solution of $4 \mathrm{sU}$ should be kept away from light. Transcription and cell growth is

restarted by adding $10 \mathrm{X}$ rich medium to the culture ( $5 \mathrm{~g}$ tryptone, $2.5 \mathrm{~g}$ yeast extract, $15 \mathrm{~g}$ $\mathrm{Na}_{2} \mathrm{HPO}_{4}, 8.25 \mathrm{~g} \mathrm{KH}_{2} \mathrm{PO}_{4}$ in $50 \mathrm{~mL}$ ). The culture is grown with shaking for the desired period before treatment with DMS or hydroxyl radical as described below. 


\subsubsection{Considerations for RNA metabolic labeling.}

The above protocol is optimized for footprinting rRNA in E. coli strain MRE600. When adapting the protocol to other target RNAs or bacterial strains, the following parameters should be considered: First, the duration of starvation, mechanism of RNA induction, and concentration of $4 \mathrm{sU}$, should be optimized for the specific strain and RNA. On the one hand, starvation must be long enough that precursor rRNA is no longer present, which requires 30-60 min in minimal medium in our hands. On the other hand, the period of starvation should be less than $12 \mathrm{~h}$, to avoid activating scavenging ribonucleases that reduce the quality of the RNA. A further consideration is that not all cells readily take up 4sU. In some cases, it is necessary to overexpress a nucleoside transporter in order to accumulate a sufficient amount of $4 \mathrm{sU}$ in the cell [16].

The appropriate growth conditions can be determined by starving cells for different periods of time before nutrient upshift (Figure 2). We analyzed rRNA processing, yield and quality by extension of a complementary ${ }^{32} \mathrm{P}$-labeled primer. We determine the appropriate concentration of $4 \mathrm{sU}$ in a similar fashion, by varying the concentration of $4 \mathrm{sU}$ added before nutrient upshift. In this case, the labeled RNA is pulled down with streptavidin beads as described below, and the wash and elution fractions analyzed by primer extension to compare the recovery of pre-rRNA and mature RNA. The ideal $4 \mathrm{sU}$ concentration is the minimal amount at which the pre-rRNA is found almost exclusively in the eluted fraction. 


\subsection{Beamline parameters for X-ray-dependent Hydroxyl Radical Footprinting}

Unlike the in vitro experiments, in which hydroxyl radicals are produced using $\mathrm{Fe}(\mathrm{II})-\mathrm{EDTA}$ and millimolar concentration of $\mathrm{H}_{2} \mathrm{O}_{2}$ [39-41], the live cell samples are probed by hydroxyl radicals that are generated by ionizing radiation, as shown in the equation below.

$$
\mathrm{H}_{2} \mathrm{O} \stackrel{h v}{\longrightarrow} \mathrm{H}_{2} \mathrm{O}^{+}+\mathrm{e}_{\mathrm{aq}}^{-} \stackrel{\mathrm{H}_{2} \mathrm{O}}{\longrightarrow} \mathrm{H}_{3} \mathrm{O}^{+}+\cdot \mathrm{OH}+\mathrm{e}_{\mathrm{aq}}^{-}
$$

This in situ method of generating hydroxyl radical avoids the deleterious effect of hydrogen peroxide on live cells. Both $\gamma$-rays from a ${ }^{137}$ Cs source [25] and X-rays from a synchrotron source [42-44] produce hydroxyl radicals in situ. However, the higher flux density of a synchrotron beam delivers the necessary dose in a much shorter time, typically in less than 100 milliseconds for frozen cells [26] and 10-20 ms for liquid culture, and ensures the least perturbation to live cells. Thus, access to a synchrotron Xray source suitable for footprinting is highly recommended for this protocol. We used two beamlines for our experiments: X28C at the National Synchrotron Light Source (NSLS) at Brookhaven National Laboratory and 5.3.1 at the Advanced Light Source (ALS) at Lawrence Berkeley National Laboratory. Future experiments will be performed at beamline XFP (17-BM) at NSLS-II.

Synchrotron beamlines suitable for footprinting should provide a high flux density incident on the sample, and ideally a variable beam size ranging from $0.1 \mathrm{~mm}$ to several millimeters [43]. In general, a focusing mirror is necessary to achieve a variably sized beam [42]. The energy of the beam should allow a significant fraction of the photons to be absorbed as uniformly as possibly throughout the depth of the sample; typically, this is achieved using a broadband beam with energies in the $5-20 \mathrm{keV}$ range. A beam flux of > 
$10^{16}$ photons/sec is desirable; high flux combined with focusing ability allows for a high brightness beam, which means that shorter exposure times can be used. As with the NSLS beamline X28C [45], a facility dedicated to x-ray footprinting, a footprinting beamline should also be equipped with sample-handling apparatuses for a variety of sample configurations and environments, including both frozen $\left(-34{ }^{\circ} \mathrm{C}\right)$ and liquid samples. At X28C, we used a cooled multi-sample holder to irradiate frozen cells with exposure time controlled by electronic shutter (typically 10 to $100 \mathrm{~ms}$ ) [26] and controlled the X-ray dose to buffered samples or liquid culture by adjusting the flow rate through a capillary tube [43].

Although the NSLS is no longer operating, facilities optimized and configured for X-ray footprinting are currently under construction at NSLS-II. In the interim, a functional subset of the live cell culture exposure apparatus has been transplanted for use at ALS beamline 5.3.1. Currently, the ALS synchrotron supports two beamlines for X-ray footprinting, beamlines 5.3.1 and 3.2.1, with a collaboration-based user program in place. Access to the beamlines can be obtained through the User Office of the respective synchrotron facility, typically through the general user program. Prospective users should begin to plan their visit several months in advance of the intended experiment.

Beamline 5.3.1 is located on a bending magnet source and equipped with a platinum-coated toroidal focusing mirror suitable for focusing a white-light X-ray beam [44]. The broadband X-ray beam $(1-13 \mathrm{keV})$ exits from the beryllium window of the beampipe under ultrahigh vacuum, with a flux of $\sim 1 \times 10^{16}$ photons $/ \mathrm{sec}$. For the in vivo studies described here, the focused beam sizes were set to deliver the highest flux density 
possible which also maintained homogeneous beam over the 540 and $700 \mu \mathrm{m}$ ID microcapillary tubes used to irradiate cell samples as described in the next section.

\subsection{Hydroxyl Radical Footprinting using Live Culture}

A basic protocol for exposing live bacterial culture to a synchrotron X-ray beam is described below, using a simple apparatus such as the one shown in Figure 3. In order to probe newly synthesized rRNA in E. coli, cultures should be grown in minimal medium to stop pre-rRNA transcription as described in Section 2.2, and then fed immediately before footprinting to reactivate transcription (Figure 1). Liquid culture irradiation was performed using a custom capillary holder and slit assembly capable of being water-cooled [43]. As the culture is pumped past the X-ray beam, fractions are collected at different time points.

We culture MRE600 cells in minimal medium and dope with 4sU as described in Section 2.2. At the start of the footprinting experiment, the cell culture is transferred to a $37^{\circ} \mathrm{C}$ water bath in the beamline experiment end-station enclosure ("hutch"). During this transfer, we collect two $0.75 \mathrm{~mL}$ fractions to serve as "No Hutch" controls. These are used to determine the amount of background signal that can be attributed to sources outside the beamline enclosure. We use a magnetic stirrer to aerate and mix the culture during the footprinting experiment.

The cells are exposed to the synchrotron X-ray beam by pumping the culture through a flow cell placed in the path of the beam. We use an M50 pump (Vici) because it provides a continuous flow (up to $5 \mathrm{~mL} / \mathrm{min}$ ) and can pump an unlimited volume. We run silica tubing from the pump inlet to the bottom of the culture flask, taking care not to 
aspirate air into the flow path. A second piece of silica tubing connects the M50 pump outlet with the flow cell and fraction collector. For the flow cell, we use silica tubing between 0.54 and $0.7 \mathrm{~mm}$ internal diameter. To obtain uniform cleavage of intracellular RNA, it is necessary for the X-ray beam to cover the entire internal diameter of the flow cell tubing with as homogenous an intensity profile as possible. To achieve high levels of cleavage, the beam should be focused to the smallest area (cross-section) that still covers the sample.

To induce outgrowth and pre-rRNA transcription, 10X rich medium (5 $\mathrm{g}$ tryptone, $2.5 \mathrm{~g}$ yeast extract, $15 \mathrm{~g} \mathrm{Na}_{2} \mathrm{HPO}_{4}, 8.25 \mathrm{~g} \mathrm{KH}_{2} \mathrm{PO}_{4}$ in $50 \mathrm{~mL}$ ) is added to the starved culture using a dispensing syringe pump. The syringe pump is first primed with the 10X rich medium and the outlet connected to the culture flask (Figure 3). The fraction collector should be filled with at least 602 -mL screw-cap microcentrifuge tubes. Each microcentrifuge tube should contain $1 \mathrm{~mL}$ RNAprotect Bacteria Reagent (Qiagen) or an equivalent RNA stabilization solution. This will protect the RNA from being degraded during isolation. This ratio of RNAprotect to culture provides optimal RNA quality and quantity (data not shown).

After the culture flask, flow cell, feed tube, and fraction collector are properly configured, the experimenter exits the beamline enclosure and activates the interlock system. The pumps, fraction collector, and beam shutter should be interfaced with a computer outside the enclosure and controlled by a computer macro in LabView or similar program [45]. The instruments are programmed to collect two $0.75 \mathrm{~mL}$ fractions of 4sU-containing culture before opening the beam shutter ("Pre-Food, No Dose"). Next, the shutter is opened, and two fractions in which the starving cells are exposed to the X- 
ray beam ("Pre-Food") are collected. These fractions will serve as controls for the RNA cleavage pattern in the starved cells. After these control samples are collected, the M50 pump and fraction collector are stopped, and the beam shutter is closed. During this time, food is added to the culture flask using the syringe pump, and mixed for $10 \mathrm{~s}$ to induce pre-rRNA synthesis. After inducer is added, we open the shutter, restart the pump and fraction collector, and collect $520.75-\mathrm{mL}$ fractions at $5 \mathrm{~mL} / \mathrm{min}$ (9 s per fraction).

At the end of the run, we collect two $0.75 \mathrm{~mL}$ samples from the culture flask ("Post-Food, No Dose"). These controls test for stray radiation in the experiment endstation enclosure. If the RNA extracted from the unexposed culture at the end of the run is significantly more cleaved than RNA from unexposed controls collected at the beginning of the run, the cells were likely exposed to radiation throughout the experiment and not just at the appointed times. In this case, the run should be discarded and additional shielding should be added at the beamline to protect the culture outside the flow cell from radiation.

After the run, the tubes are capped, inverted to mix the culture with the RNAProtect, and placed in a microcentrifuge to pellet the cells. After decanting the supernatant, the cell pellets are immediately placed on dry ice, and stored at $-80{ }^{\circ} \mathrm{C}$. These final steps should be completed as promptly as possible to reduce background RNA degradation.

\subsubsection{Considerations for rRNA Quality}

The quality of the footprinting results depends greatly on minimizing X-rayindependent RNA cleavage, which leads to undesired background in the sequence 
analysis. MRE600 cells lack RNase I, a periplasmic protein that can degrade RNA during extraction [46]. To further limit non-specific RNA degradation, we use RNAprotect Bacteria Reagent (Qiagen) to stabilize the RNA. We obtain the best results when the culture and RNAprotect are mixed as soon as possible after each fraction is collected. If the samples will be shipped to another location for analysis, they should be packaged in ample dry ice to guard against partial thawing during shipment. Cells should be stored at $-80{ }^{\circ} \mathrm{C}$ until the RNA can be extracted.

\subsubsection{Considerations for rRNA Yield}

In log phase cells, nascent rRNA represents $2-5 \%$ of the total rRNA [6]. In cells emerging from starvation, nascent rRNA only represents $1-2 \%$ of the total rRNA for the first 5 minutes of outgrowth (data not shown). Downstream analysis of the 16S rRNA typically requires $4 \mu \mathrm{g}$ purified RNA to achieve full sequence coverage by random priming and high-throughput sequencing, or $10 \mu \mathrm{g}$ RNA for gene-specific priming. Therefore, every effort should be made to obtain the highest RNA yield possible. At the beamline, the volume of cells collected in each sample is limited by the flow rate ( 5 $\mathrm{mL} / \mathrm{min}$ for the M50 pump) and desired time resolution ( $9 \mathrm{~s}$ per $0.75 \mathrm{~mL}$ fraction). Each $0.75 \mathrm{~mL}$ fraction typically yields $\sim 60-150 \mathrm{ng}$ purified $4 \mathrm{sU}-\mathrm{RNA}$, although this depends on the cell density and the extent of labeling. If RNA other than rRNA is to be probed, the total amount of sample required will depend on the abundance of the transcript of interest. The RNA yield can be improved by increasing the RNA overexpression, collecting larger fractions, or examining the RNA at steady-state, eliminating the need for 4sU labeling. For RNAs $\leq 300 \mathrm{nt}$, full sequence coverage can be achieved using less 
material. If only one gene-specific primer is required, only $500 \mathrm{ng}$ purified RNA is sufficient.

\subsection{Obtaining the proper $X$-ray dose}

For reliable analysis of the hydroxyl radical cleavage pattern by primer extension, the RNA should be cleaved no more than once within each detection window [47]. If an RNA strand is cleaved multiple times, those events nearer to the location of priming will be preferentially detected over events further from the site of priming. Thus, for analysis by traditional gene-specific priming, the X-ray dose should be adjusted such that 70-90\% of the RNA is full-length. Assuming the cleavage events follow a Poisson distribution, if $80 \%$ of the RNA is uncleaved, $\sim 19 \%$ will be cleaved once, and the remaining $~ 1 \%$ will be cleaved twice. If high-throughput sequencing is used to analyze the footprinting pattern, the RNA is typically fragmented to $\sim 200$ nucleotides before library construction [37]. In this instance, a much higher X-ray dose, sufficient to cleave the RNA once per 200 nucleotides, is needed to ensure good signal-to-noise.

To determine the correct X-ray exposure, we generate a dose-response curve by placing aluminum sheets of different thickness in front of the flow cell to attenuate the beam intensity incident on the sample. Alternatively, the X-ray dose is varied by changing the sample flow rate, which alters the time needed for the sample to traverse the beam. After exposing the cells to the X-ray beam, the amount of full-length 16S RNA remaining in each sample is determined by isolating total RNA, and extending a radiolabeled primer complementary to nucleotides $1486-1510$ at the 3 '-end of the $16 \mathrm{~S}$ rRNA. The percent full-length cDNA (normalized to an unexposed sample) is plotted 
versus the aluminum thickness, and fit to a line. This dose-response plot is used to empirically determine the appropriate beam attenuation needed to obtain the desired level of cleavage (Figure 4).

A few methods have been devised to estimate the intensity of the beam prior to each run. The first is a fluorescence-based assay using Alexa-488 [45], which is photobleached upon exposure to the X-ray beam. A $2 \mu \mathrm{M}$ solution of Alexa-488 is pumped past the beam at a range of flow rates (i.e. different exposure times), with or without aluminum attenuation. The exposed Alexa-488 solution is collected, diluted into the linear range of the fluorimeter, and the fluorescence intensity measured. The rate constants for quenching Alexa-488 obtained for the different attenuations can be compared between runs. This information is used to adjust the attenuation to obtain the appropriate dosage.

A second method for rapidly estimating the $\mathrm{X}$-ray dose at the beamline is to expose log-phase culture to the X-ray beam using different thicknesses of aluminum to attenuate the beam. After extracting the total RNA, a primer complementary to the 3'-end of the RNA is extended using reverse transcriptase. The RNA is degraded with RNase H. The cDNA is then analyzed by real time qPCR (Quantitect SYBR Green PCR Kit, Qiagen) to determine the amounts of full-length RNA relative to an unexposed sample. Although not as accurate as extension of a radiolabeled primer, the qPCR results can be used to confirm that a given experimental configuration will yield an appropriate cleavage rate. 


\subsection{Footprinting rRNA in live culture using DMS}

To probe the rRNA secondary structure with dimethyl sulfate (DMS), we adapted the in vivo DMS probing and quenching conditions of Wells et al. [48] to our protocol. For a starvation-recovery time course, MRE600 cells are cultured as described in Section 2.2. The volume of each sample needed to recover sufficient RNA for the intended sequencing analysis should be determined in advance (see above). This amount should be multiplied by the desired number of time points and controls to determine the total culture volume required for the experiment. Ideally, one should collect control samples to which no DMS is added for each time point to be assayed. In practice, the no DMS background changes slowly during outgrowth, so it is sufficient to collect an untreated control every few minutes.

For each sample to be treated with DMS, the desired amount of DMS diluted 1:4 $(\mathrm{v} / \mathrm{v})$ in $95 \%$ ethanol is placed in a $50 \mathrm{~mL}$ screw-cap conical tube. DMS is toxic and mutagenic; place tubes in a chemical fumehood if available, wear eye shields and protective gloves and clothing, and avoid inhalation or contact with the skin. Ethanol interferes with phospholipid bilayers, so the amount of DMS and ethanol added to samples should be as low as possible while still giving sufficient probing. The ratio of DMS-ethanol solution to culture medium was typically 1:25 (v/v) in these experiments.

At the desired times, an aliquot (e.g. $20 \mathrm{~mL}$ ) is removed from the main culture and added to one of the $50 \mathrm{~mL}$ tubes. After 30 seconds, the methylation reaction is quenched with the addition of a $1 / 2$ volume (e.g. $10 \mathrm{~mL}$ ) of ice-cold $0.6 \mathrm{M} \beta$ mercaptoethanol and a 1/2 volume of water-saturated isoamyl alcohol [48]. 
After DMS treatment, pellet the cells in each sample by centrifugation and resuspend the cells in one culture volume (e.g. $20 \mathrm{~mL}$ ) $0.6 \mathrm{M} \beta$-mercaptoethanol [48]. Addition of RNAprotect (Qiagen) is unnecessary. Harvest the cells again and decant the supernatant. At this point, continue with RNA extraction, or store the cell pellets at -80 ${ }^{\circ} \mathrm{C}$ until ready to extract the RNA.

\subsubsection{Optimizing the Amount of DMS}

The amount of DMS to be used should be determined from a dose response curve as described for hydroxyl radical footprinting (more below). For consistent results, it is essential that all samples be treated with DMS for the same amount of time. The time should be determined based on the desired time resolution of the experiment.

\subsection{Isolation of 4sU-labeled rRNA from E. coli}

Structural analysis of probed $16 \mathrm{~S}$ rRNA requires $4-10 \mu \mathrm{g}$ of high-quality RNA. We use RNeasy spin columns (Qiagen) to extract total RNA from treated bacterial cell pellets. For a smaller number of samples with larger volume, alternative RNA isolation methods such as Trizol may be less costly and similarly effective.

To isolate nascent 4sU-labeled RNA, 4sU is first biotinylated and then captured on streptavidin beads (Figure 5). The purification protocol that follows is based on that of Dölken et al [17] with slight modifications to the buffers and the incubation durations.

The 4sU-labeled RNA is modified using EZ-Link HPDP-Biotin (Pierce), freshly dissolved in dimethylformamide to a concentration of $2.2 \mathrm{mg} / \mathrm{mL}$. We dilute the RNA to 
$100 \mathrm{ng} / \mu \mathrm{L}$ in $10 \mathrm{mM}$ Tris-HCl pH 7.6, $1 \mathrm{mM}$ EDTA, and add the HPDP-Biotin reagent to a final concentration of $0.4 \mathrm{mg} / \mathrm{mL}$, for $10 \mathrm{~min}$ at $65^{\circ} \mathrm{C}$.

Because unreacted biotin can interfere with affinity purification in the next step, we remove unbound biotin by extracting the biotinylation reaction with an equal volume of chloroform/isoamyl alcohol (39:1). The upper aqueous layer is collected using Phaselock-gel (Heavy) tubes (Eppendorf). The RNA (200-300 $\mu \mathrm{L})$ is precipitated by adding $1 / 10$ volume $3 \mathrm{M}$ sodium acetate ( $\mathrm{pH}$ 5.5) and 3 volumes $100 \%$ ethanol, incubating $1 \mathrm{~h}$ at $-80{ }^{\circ} \mathrm{C}$, and pelleted by centrifugation at $13,000 \mathrm{x} g$ at $4{ }^{\circ} \mathrm{C}$ for $30 \mathrm{~min}$. The pellet is washed with 1 volume $75 \%$ ethanol, centrifuged again for $10 \mathrm{~min}$, and dried under vacuum. The pellet is resuspended in $100 \mu \mathrm{L}$ binding buffer $(20 \mathrm{mM}$ Tris- $\mathrm{HCl} \mathrm{pH}$ 7.6, 1 mM EDTA, $0.5 \mathrm{M} \mathrm{NaCl}$ ) before determining the RNA concentration (Nanodrop UV spectrometer).

The resuspended biotinylated RNA $(50-150 \mu \mathrm{g})$ is added to $100-150 \mu \mathrm{L}$ magnetic streptavidin beads (NEB) for $1 \mathrm{~h}$ at $4{ }^{\circ} \mathrm{C}$ with gentle shaking. Before adding the RNA, the beads should be washed three times with binding buffer, blocked with $40 \mathrm{ng} / \mathrm{mL}$ glycogen in binding buffer, then washed once more with binding buffer. After the supernatant-containing unlabeled RNA is removed, we wash the beads three times with one volume wash buffer (100 mM Tris- $\mathrm{HCl}$ pH 7.6, $10 \mathrm{mM}$ EDTA, $0.5 \mathrm{M} \mathrm{NaCl}, 0.5 \%$ Tween20) at $65^{\circ} \mathrm{C}$ and three more times with wash buffer at room temperature. The supernatant containing unlabeled RNA and first wash may be combined and saved.

The labeled RNA is eluted from the beads using one volume freshly prepared 100 $\mathrm{mM}$ dithiothreitol (DTT). This elution step is repeated, and the eluates combined. The RNA is precipitated from the eluate using 1/10 volume $3 \mathrm{M}$ sodium acetate, three 
volumes $100 \%$ ethanol, and $40 \mathrm{ng} / \mathrm{mL}$ glycogen as a carrier. The RNA pellet is collected and washed as described above. The RNA is resuspended in $25 \mu \mathrm{L}$ RNase-free water and the concentration determined by UV absorption. If desired, the washes containing the unlabeled RNA can also be purified in the same manner. Alternatively, the RNA may be purified using RNeasy MinElute Spin columns.

The yield of 4sU-labeled RNA should be $1-2 \%$ of the input and reflects the extent of $4 \mathrm{sU}$ incorporation under these conditions. We analyze the quality of 4 sU-labeled RNA by extending a ${ }^{32} \mathrm{P}$ - or dye-labeled primer on RNA recovered from the wash and elution fractions. In this example, the pre-rRNA should be primarily found in the eluted fraction (Figure 6). Alternatively, RNA transcribed in vitro in the presence of 4S-UTP can be used as an internal standard to validate the purification procedure. Although we expect very little DNA contamination with this procedure, the total RNA can be treated with RNase-free DNase if residual DNA is a concern.

\subsection{Analysis of 4 sU-labeled RNA}

Footprinting patterns have historically been analyzed by extending gene-specific primers by reverse transcriptase and examining the cDNA that is produced $[49,50]$. This can be done with radiolabeled primers, in which case, the cDNAs are separated on a traditional sequencing gel and analyzed by autoradiography. Each primer requires $0.5-1$ $\mu \mathrm{g}$ RNA and can be expected to give sequence data for roughly 200 nucleotides. The band intensities in the gel can be analyzed using the Semi-Automated Footprinting Analysis (SAFA) software package [51]. Footprinting analysis can also be performed by extending fluorophore-labeled primers and separating the resulting cDNA using capillary 
electrophoresis [52]. Each primer requires $0.5 \mu \mathrm{g}$ RNA and can be expected to give sequence data for 300-400 nucleotides. Capillary electrophoresis data can be analyzed using ShapeFinder [53]. A further possibility for analysis is to use high-throughput sequencing [37,54]. This technique does not carry with it the need for gene-specific priming. These methods are all extensively described in the literature and will not be discussed further here.

\section{Example: Probing the 3' domain of $16 \mathrm{~S}$ rRNA during assembly}

We conclude this protocol with an example showing how in vivo DMS probing can be used to generate structural data on in vivo ribosome assembly intermediates. A $220 \mathrm{~mL}$ culture of phosphate-starved cells was pulse-labeled with $4 \mathrm{sU}$ to a final concentration of $0.5 \mathrm{mM}$ and probed with DMS at time points before and during outgrowth. A total of $1415-\mathrm{mL}$ time points and controls were collected. Samples were treated with DMS immediately before the food was added to start outgrowth, and then over the first 8 min after feeding. No DMS controls were collected immediately before feeding, and at 4 min and 8 min after feeding. The 4sU RNA was isolated as described above, and the methylation patterns were analyzed by traditional primer extension sequencing, using primers complementary to the 16S rRNA (Figure 7a). Band intensities were analyzed using SAFA (Figure 7b).

Our initial results using this technique are consistent with earlier findings regarding the folding of the central platform and 3' domain of the 30S ribosome (Figure 7c). For example, nucleotides $890-920$ in the central domain are strongly exposed to methylation in the newly transcribed 4sU-labeled pre-rRNA. Nucleotides 885-912 form 
helix 27, which supports the decoding site helix 44. Nucleotides 917-919 form part of the central pseudoknot, a structure that draws together the 5', central, and 3' domains and that is known to form late in $30 \mathrm{~S}$ assembly [55]. Also prominently exposed in the $17 \mathrm{~S}$ pre-rRNA is helix 33, which forms the "beak" of the 30S ribosome and is known to fold late in assembly [55]. More surprisingly, certain nucleotides in the 5' domain also take about 6 minutes to become fully protected, although this region is the first domain to be transcribed. Thus, this method can pinpoint RNA interactions that form late during assembly of the 30S ribosomal subunit, or that lie on the interface with 50S subunits.

\section{Conclusions}

This method of isolating nascent RNA over time, combined with the in vivo RNA probing techniques of hydroxyl radical and DMS footprinting, will allow the kinetics of structural changes in rRNA during ribosome biogenesis to be determined. This is a novel way of examining ribosome assembly intermediates that does not require the use of temperature-sensitive cells strains or conditional mutants. The time resolution currently attainable with these probing methods is appropriate for the study of ribosome biogenesis, and may be improved in the future. This technique has the potential to heighten our understanding of ribosome biogenesis in ways that would not be possible by in vitro studies. For example, this method would be very conducive to studies of ribosome assembly factors in vivo. By using cell strains lacking ribosome assembly factors, it should be possible to use this technique to determine, not just where these proteins interact with the assembling ribosome, but when they interact as well. Finally, 
this method can be adapted to map time-dependent conformational changes in a wide range of stable, non-coding RNAs.

\section{Acknowledgments}

The authors thank Don Abel, Rich Celestre, and John Toomey for technical assistance. This work was supported by a grant from the National Institutes of Health (R01 GM60819 to S.A.W.). The Advanced Light Source is supported by the Director, Office of Science, Office of Basic Energy Sciences, of the U.S. Department of Energy under Contract No. DE-AC02-05CH11231. The National Synchrotron Light Source, Brookhaven National Laboratory, was supported by the U.S. Department of Energy, Office of Science, Office of Basic Energy Sciences, under Contract No. DE-AC0298CH10886. The Center for Synchrotron Biosciences at the National Synchrotron Light Sources is supported by NIBIB under P30-EB0966, with research instrumentation development supported by the National Science Foundation under DBI-1228549.

\section{References}

[1] S.A. Mortimer, M.A. Kidwell, J.A. Doudna, Nat. Rev. Genet. 15 (2014) 469-79.

[2] M. Kubota, C. Tran, R.C. Spitale, Nat. Chem. Biol. 11 (2015) 933-941.

[3] T. Powers, G. Daubresse, H.F. Noller, J. Mol. Biol. 232 (1993) 362-74.

[4] S. Spillmann, F. Dohme, K.H. Nierhaus, J. Mol. Biol. 115 (1977) 513-23.

[5] B.T. Lewicki, T. Margus, J. Remme, K.H. Nierhaus, J. Mol. Biol. 231 (1993) 58193.

[6] L. Lindahl, J Mol Biol 92 (1975) 15-37. 
[7] D.N. Wilson, K.H. Nierhaus, Crit. Rev. Biochem. Mol. Biol. 42 187-219.

[8] M. Kaczanowska, M. Rydén-Aulin, Microbiol. Mol. Biol. Rev. 71 (2007) 477-94.

[9] S.S. Chen, E. Sperling, J.M. Silverman, J.H. Davis, J.R. Williamson, Mol. Biosyst. 8 (2012) 3325-34.

[10] A. El Hage, D. Tollervey, RNA Biol. 1 (2014) 9-14.

[11] W.A. Held, B. Ballou, S. Mizushima, M. Nomura, J. Biol. Chem. 249 (1974) $3103-11$.

[12] K.H. Nierhaus, F. Dohme, Proc. Natl. Acad. Sci. U. S. A. 71 (1974) 4713-7.

[13] G.M. Culver, Biopolymers 68 (2003) 234-49.

[14] S.S. Chen, J.R. Williamson, J. Mol. Biol. 425 (2013) 767-79.

[15] H.D. Murray, D.A. Schneider, R.L. Gourse, Mol. Cell 12 (2003) 125-34.

[16] A. Favre, R. Bezerra, E. Hajnsdorf, Y. Lemaigre Dubreuil, A. Expert-Bezancon, Eur J Biochem 160 (1986) 441-449.

[17] L. Dölken, Z. Ruzsics, B. Rädle, C.C. Friedel, R. Zimmer, J. Mages, R. Hoffmann, P. Dickinson, T. Forster, P. Ghazal, U.H. Koszinowski, RNA 14 (2008) 1959-72.

[18] J. Spitzer, M. Hafner, M. Landthaler, M. Ascano, T. Farazi, G. Wardle, J. Nusbaum, M. Khorshid, L. Burger, M. Zavolan, T. Tuschl, Methods Enzymol. 539 (2014) 113-61.

[19] L. Gay, K. V Karfilis, M.R. Miller, C.Q. Doe, K. Stankunas, Nat. Protoc. 9 (2014) 410-20.

[20] S. Stern, D. Moazed, H.F. Noller, Ribosomes, Elsevier, 1988.

[21] C. Brunel, P. Romby, RNA-Ligand Interactions Part B, Elsevier, 2000.

[22] A. Ephrussi, G.M. Church, S. Tonegawa, W. Gilbert, Science 227 (1985) 134-40. 
[23] C. Waldsich, R. Grossberger, R. Schroeder, Genes Dev. 16 (2002) 2300-12.

[24] J.J. Hayes, L. Kam, T.D. Tullius, Methods Enzymol. 186 (1990) 545-9.

[25] L.M. Ottinger, T.D. Tullius, J. Am. Chem. Soc. 122 (2000) 5901-5902.

[26] T. Adilakshmi, S.F. Soper, S.A. Woodson, Methods Enzym. 468 (2009) 239-258.

[27] T. Adilakshmi, D.L. Bellur, S.A. Woodson, Nature 455 (2008) 1268-1272.

[28] B. Sclavi, M. Sullivan, M.R. Chance, M. Brenowitz, S.A. Woodson, Science 279 (1998) 1940-3.

[29] S.F. Clatterbuck Soper, R.P. Dator, P.A. Limbach, S.A. Woodson, Mol. Cell 52 (2013) 506-16.

[30] C.Y. Ralston, B. Sclavi, M. Sullivan, M.L. Deras, S.A. Woodson, M.R. Chance, M. Brenowitz, Methods Enzymol. 317 (2000) 353-68.

[31] T.D. Tullius, J.A. Greenbaum, Curr. Opin. Chem. Biol. 9 (2005) 127-34.

[32] S. Stern, T. Powers, L.M. Changchien, H.F. Noller, Science 244 (1989) 783-90.

[33] C. Merryman, D. Moazed, G. Daubresse, H.F. Noller, J. Mol. Biol. 285 (1999) $107-13$.

[34] T. Nguyenle, M. Laurberg, M. Brenowitz, H.F. Noller, J. Mol. Biol. 359 (2006) $1235-48$.

[35] T. Powers, H.F. Noller, RNA 1 (1995) 194-209.

[36] S. Rouskin, M. Zubradt, S. Washietl, M. Kellis, J.S. Weissman, Nature 505 (2014) $701-5$.

[37] J. Talkish, G. May, Y. Lin, J.L. Woolford, C.J. McManus, RNA 20 (2014) 713-20.

[38] A. Swiatkowska, W. Wlotzka, A. Tuck, J.D. Barrass, J.D. Beggs, D. Tollervey, RNA 18 (2012) 2187-200. 
[39] S. UDENFRIEND, C.T. CLARK, J. AXELROD, B.B. BRODIE, J. Biol. Chem. 208 (1954) 731-9.

[40] T.D. Tullius, B.A. Dombroski, Proc. Natl. Acad. Sci. U. S. A. 83 (1986) 5469-73.

[41] T.D. Tullius, B.A. Dombroski, M.E. Churchill, L. Kam, Methods Enzymol. 155 (1987) 537-58.

[42] M.R. Sullivan, S. Rekhi, J. Bohon, S. Gupta, D. Abel, J. Toomey, M.R. Chance, Rev. Sci. Instrum. 79 (2008) 025101.

[43] J. Bohon, R. D’Mello, C. Ralston, S. Gupta, M.R. Chance, J. Synchrotron Radiat. 21 (2014) 24-31.

[44] S. Gupta, R. Celestre, C.J. Petzold, M.R. Chance, C. Ralston, J. Synchrotron Radiat. 21 (2014) 690-9.

[45] S. Gupta, M. Sullivan, J. Toomey, J. Kiselar, M.R. Chance, J. Synchrotron Radiat. 14 (2007) 233-43.

[46] M.P. Deutscher, in:, C. Ciaran (Ed.), Prog Mol Biol Transl Sci, Academic Press, 2009, pp. 369-391.

[47] M. Hsieh, M. Brenowitz, Methods Enzymol. 274 (1996) 478-92.

[48] S.E. Wells, J.M.. Hughes, A. Haller Igel, M. Ares, RNA-Ligand Interactions Part B, Elsevier, 2000.

[49] T. Inoue, T.R. Cech, Proc. Natl. Acad. Sci. U. S. A. 82 (1985) 648-52.

[50] D. Moazed, S. Stern, H.F. Noller, J. Mol. Biol. 187 (1986) 399-416.

[51] R. Das, A. Laederach, S.M. Pearlman, D. Herschlag, R.B. Altman, RNA 11 (2005) $344-54$.

[52] T. Adilakshmi, S.F.C. Soper, S.A. Woodson, Methods Enzymol. 468 (2009) 239- 
58.

[53] S.M. Vasa, N. Guex, K.A. Wilkinson, K.M. Weeks, M.C. Giddings, RNA 14 (2008) 1979-90.

[54] L.J. Kielpinski, J. Vinther, Nucleic Acids Res. 42 (2014) e70.

[55] K.L. Holmes, G.M. Culver, J. Mol. Biol. 354 (2005) 340-57.

\section{FIGURE LEGENDS}

Figure 1. Overview of time-resolved in vivo footprinting protocol. The method consists of three major parts: (1) starvation recovery, (2) in vivo RNA footprinting, and (3) isolation and analysis of the RNA. In the first phase, pre-rRNA transcription is synchronized by starving cells of phosphate, then adding nutrients and phosphate to trigger de novo ribosome biogenesis. The modified nucleotide 4-thiouridine (4sU) is added to the media prior to food. During the recovery from starvation, 4sU-labeled nascent rRNA is probed with hydroxyl radical or DMS in the second phase. In the third phase, the 4sU-labeled RNA is isolated from harvested cells and sequenced.

Figure 2. Pre-ribosomal RNA synthesis during starvation recovery. Percent $17 \mathrm{~S}$ pre-rRNA versus time after nutrients were added to MRE600 culture grown in minimal (low phosphate) medium for $0.5,6,12$, or $18 \mathrm{~h}$ before feeding. The pre-existing prerRNA is usually depleted within 30-60 min after transfer to low phosphate medium. In this example, some pre-rRNA remains after $30 \mathrm{~min}$, accounting for the large amount of RNA at the start of the time course. The fraction of 17S rRNA was determined for each sample by primer extension, and calculated from $f=\mathrm{cpm}[17 \mathrm{~S}] /(\mathrm{cpm}[16 \mathrm{~S}]+\mathrm{cpm}$ 
$[17 \mathrm{~S}])$.

Figure 3. Apparatus for X-ray footprinting of live cultures. (a) Schematic showing the flow of liquid culture past the X-ray beam and into a fraction collector. A syringe pump (Harvard Model 33) is used to dispense nutrients into the culture at the desired time. A M50 pump (Vici) displaces culture through a capillary flow cell in the path of the $\mathrm{X}$-ray beam [43], at flow rates up to $5 \mathrm{~mL} / \mathrm{min}$. The $\mathrm{X}$-ray dose depends on the flow rate, the length of tubing exposed to the beam (horizontal slit), and the X-ray flux density of the beam itself. The exposed culture is collected in $0.75-1 \mathrm{~mL}$ fractions. (b) Photograph of apparatus at NSLS X28C. The X-ray beam pipe is pointing toward the front of the image.

Figure 4. X-ray dose-response curves for RNA footprinting. Full-length 16S rRNA remaining after exposure of log-phase MRE600 culture to an X-ray beam, using the flow apparatus described in Fig. 3. The flow rate was $5 \mathrm{~mL} / \mathrm{min}$. The capillary tubing was 700 $\mu \mathrm{m}$ ID. The fraction full-length RNA was determined by primer extension and normalized to the amount of RNA in unirradiated controls. The X-ray dose was varied by attenuating the beam with aluminum sheets of varying thickness. The curves represent experiments performed on different dates at NSLS X28C (BNL) and ALS 5.3.1.

Figure 5. Scheme for isolation of 4sU-containing RNA. Newly transcribed RNA is metabolically labeled with 4-thiouridine added to the medium just before outgrowth. 4sU 
is covalently modified with bifunctional HPDP-Biotin, which reacts with reduced thiols. The biotinylated RNA is captured on streptavidin beads, and then eluted with $0.1 \mathrm{M}$ DTT. The purified RNA is analyzed by traditional or high-throughput sequencing.

Figure 6. Analysis of 4sU-RNA purification. $6 \%$ sequencing gel (1X TBE) showing the primer extension of RNA from elution and flow-through fractions of $4 \mathrm{sU}$ affinity purification (10 $\mu \mathrm{g}$ input RNA) as outlined in Figure 5. The unlabeled rRNA will be in the flow-through fractions. The pre-rRNA should be almost exclusively in the elution fractions. The primer used for probing the 5 ' end of the $17 \mathrm{~S}$ anneals to nucleotides $162-$ 178 of the $16 \mathrm{~S}$ rRNA.

Figure 7. Time-resolved DMS footprinting of rRNA in E. coli. (a) $6 \%$ sequencing gel (1X TBE) showing the change in the primer extension pattern from $0-8$ min after the addition of food. Lanes ND indicate samples with no DMS. Sequencing standards are on the left. Examples of residues that become protected from DMS methylation with different kinetics are denoted as in (c). (b) Example of integrated band intensities for each nucleotide, for $16 \mathrm{~S}$ nucleotides 880 to 1042 . Black, no DMS treatment (4 min); blue, DMS treatment before feeding; red, DMS treatment 4 min after feeding. c) Secondary structure and 3D ribbon (2i2p) of the E. coli $16 \mathrm{~S}$ rRNA showing the folding kinetics for individual residues as the time to reach saturation (pink, red, brown); n.d. (gold), change in methylation but rate not determined. 


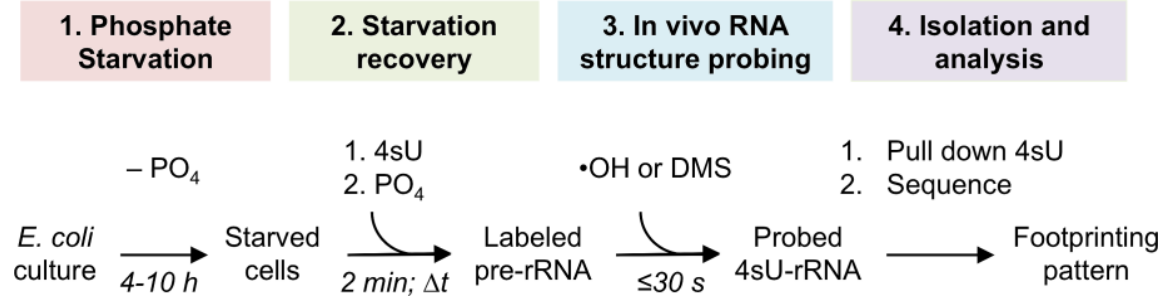

Figure 1 


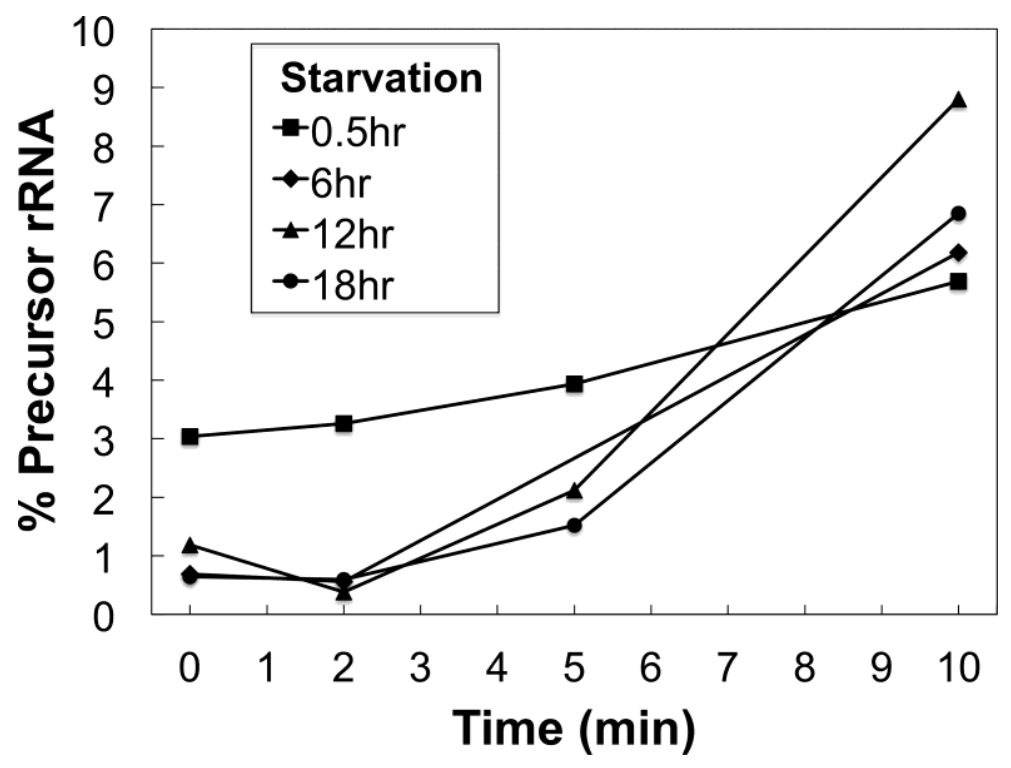

Figure 2 

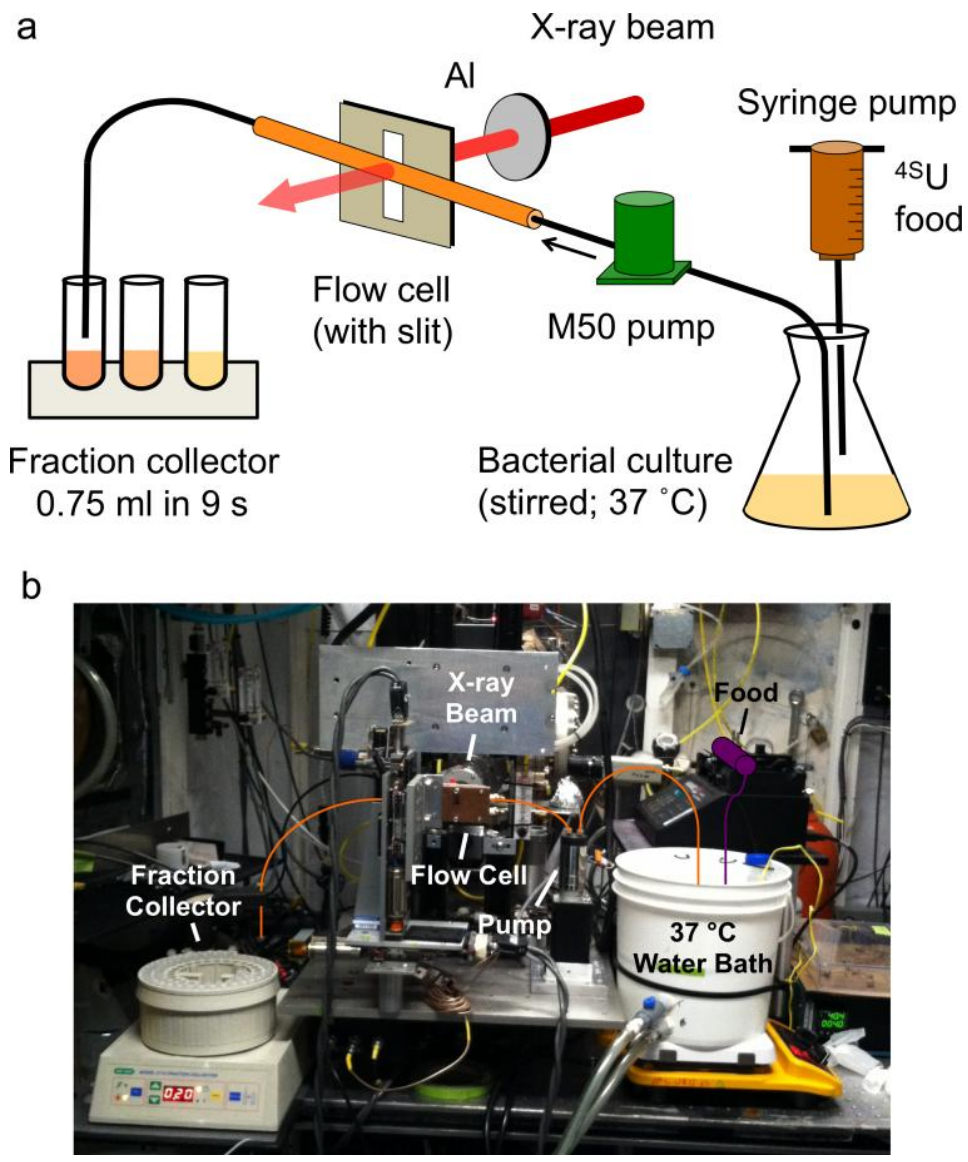

Figure 3 


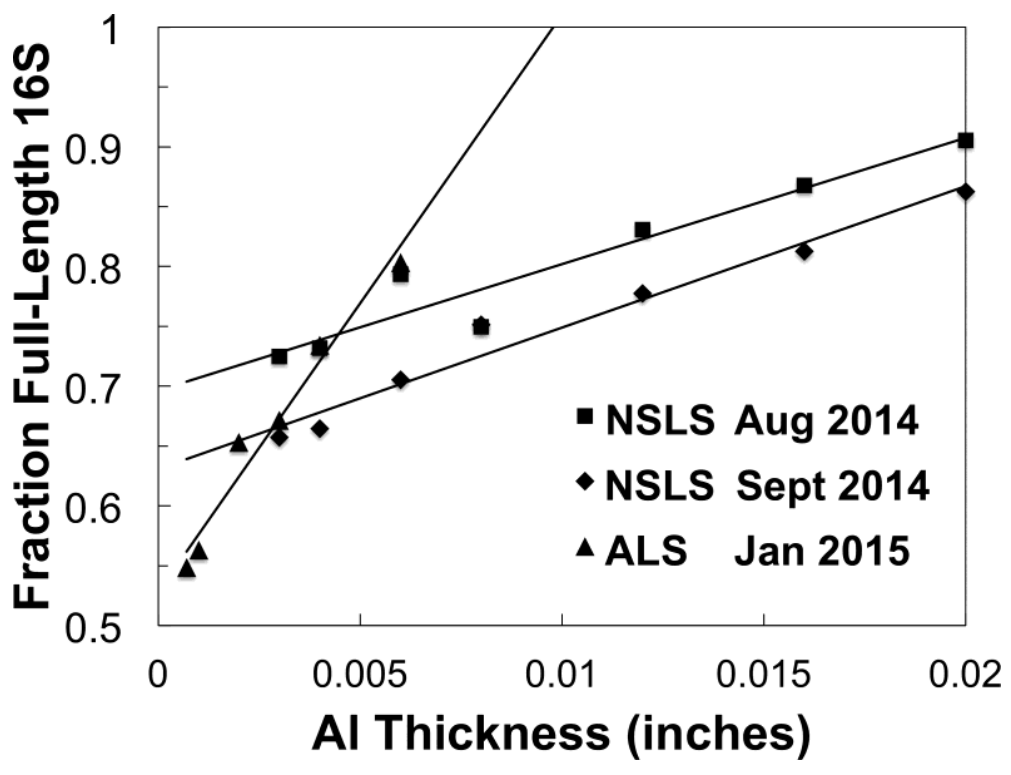

Figure 4 
1. Metabolically label nascent rRNA

2. Biotinylate
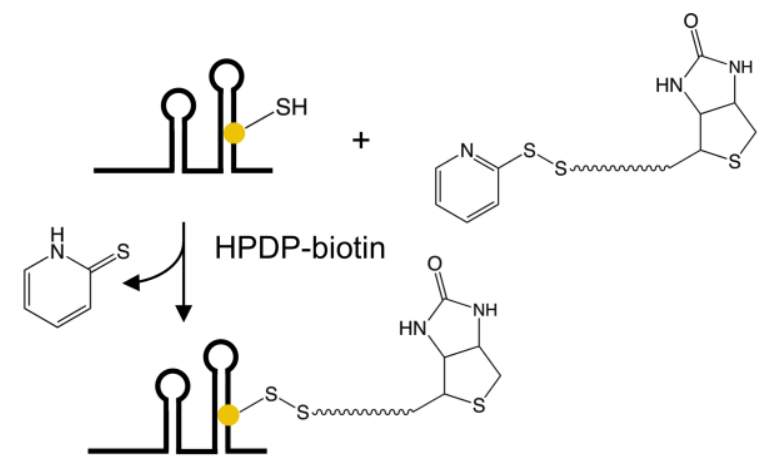

3. Capture

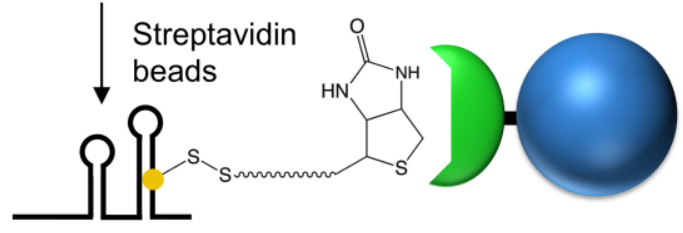

4. Elute

5. Sequence

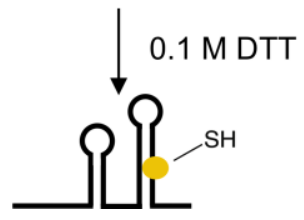

Figure 5 


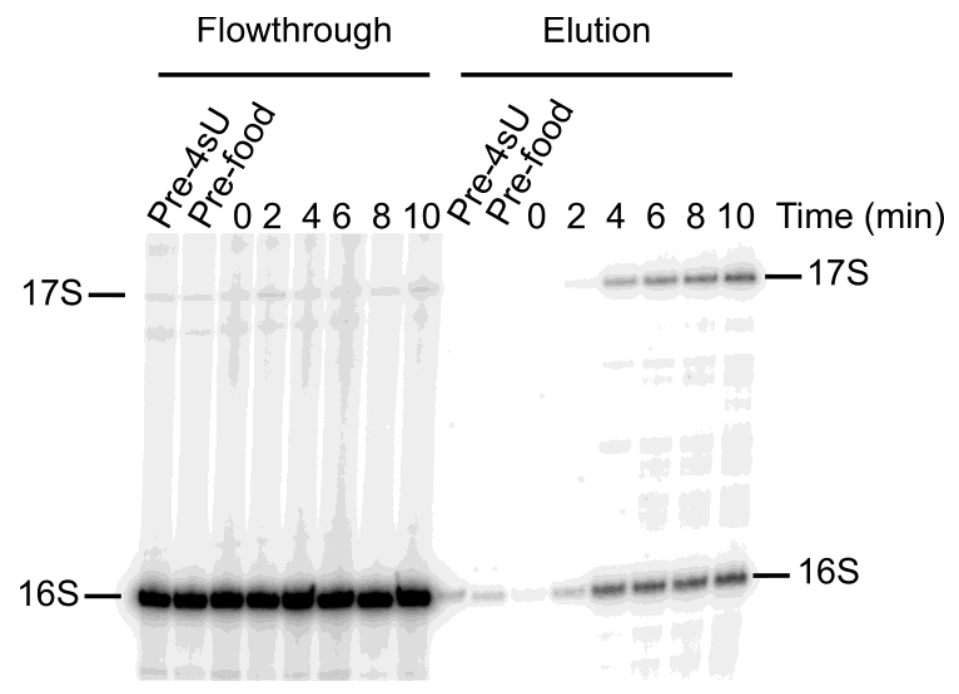

Figure 6 
a

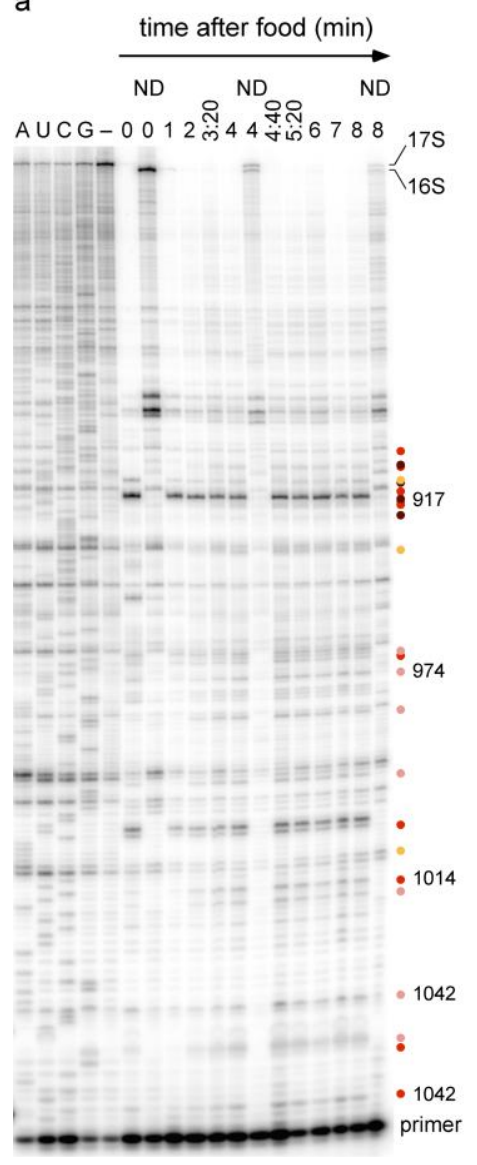

b

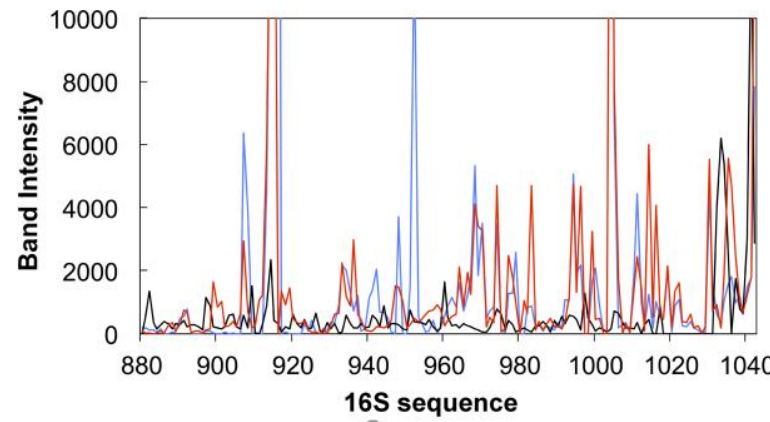

C

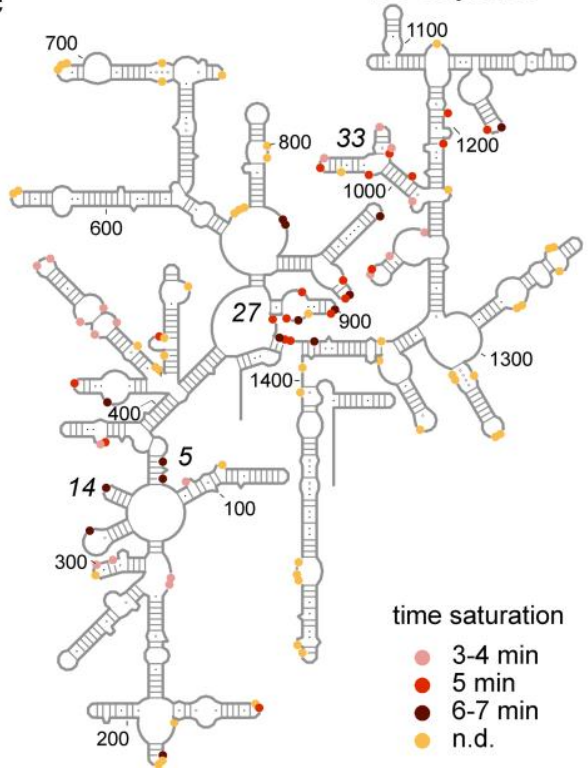

Figure 7
4 min no DMS

0 min DMS

4 min DMS

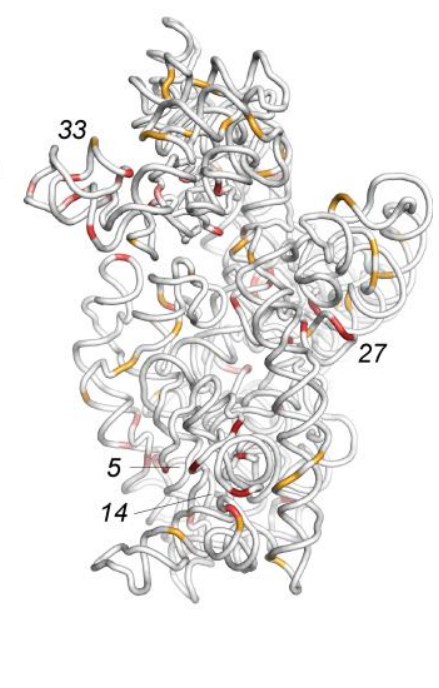

